\title{
Enhancements of pinning by superconducting nanoarrays
}

\author{
E. Navarro, ${ }^{1,2}$ C. Monton, ${ }^{1}$ J. Pereiro,,${ }^{1,3}$ Ali C. Basaran, ${ }^{1,4}$ and Ivan K. Schuller ${ }^{1}$ \\ ${ }^{1}$ Department of Physics and Center for Advanced Nanoscience, University of California San Diego, La Jolla, California 92093, USA \\ ${ }^{2}$ Departamento de Física de Materiales, Facultad de Físicas, Universidad Complutense, 28040 Madrid, Spain \\ ${ }^{3}$ School of Physics and Astronomy, Cardiff University, The Parade, Cardiff CF24 3AA, United Kingdom \\ ${ }^{4}$ Department of Physics, Gebze Technical University, Gebze, Kocaeli 41400, Turkey \\ (Received 5 June 2015; revised manuscript received 30 August 2015; published 21 October 2015)
}

\begin{abstract}
We present a comparative study of vortex pinning efficiency in superconducting (V) thin films grown on two similar triangular arrays of superconducting $(\mathrm{Nb})$ and nonsuperconducting $(\mathrm{Cu})$ nanodots. Resistance and magnetization anomalies at the same matching fields confirm the same pinning periodicity in both samples. However, we found two distinct features: First, the sample with superconducting dots shows stronger pinning that appears as sharper matching peaks in magnetization loops and shows higher critical current density and larger critical field at low temperatures. Second, an overall increase in the resistance of the $\mathrm{V}$ film with $\mathrm{Nb}$ nanodots is observed, while there is a crossover in the temperature dependence of the critical field and the critical current of both samples at $T=3 \mathrm{~K}$. This crossover corresponds to the temperature when the superconducting coherence length of $\mathrm{V}$ thin film equals the edge-to-edge distance between nanodots. We argue that this change in superconducting properties is related to the change in the superconducting regime from pinning enhancement at low temperatures to a superconducting wire network at high temperatures.
\end{abstract}

DOI: 10.1103/PhysRevB.92.144512 PACS number(s): 74.25.Wx, 74.78.Na, 74.25.Sv, 74.25.Op

\section{INTRODUCTION}

The improvement of vortex pinning efficiency in type-II superconductors has been the focus of many experimental and theoretical investigations [1]. It is well established that artificial pinning centers can substantially enhance the superconducting critical current density $\left(J_{C}\right)$ whenever the density of vortices matches the underlying pinning landscape ("pinning enhancement"). This commensurability effect between vortices and pinning landscape has been widely studied in different periodic [2] and nonperiodic [3,4] pinning geometries using pinning centers such as micrometric and submicrometric holes [5-8], blind holes $[9,10]$, and magnetic $[11,12]$ and nonmagnetic dots [13,14].

Matching effects appear as "dips" in the magnetic field dependence of the resistance, $R(H)$, and "peaks" in the field dependence of the magnetization, $M(H)$, when an integer number of flux quanta, $n \phi_{o}$, is commensurate with the unit cell of the pinning centers [5]. However, when the width $(w)$ of the superconducting (SC) strips around the dots is comparable to the SC coherence length, $\xi(T)$, the vortices are too large to locate in interstitial positions. In this case, matching also occurs at integer flux quantum fields, but the sample is described as a SC wire network [15]. These matching effects are due to the additional suppression of the $\mathrm{SC}$ critical temperature $\left(T_{C}\right)$ by magnetic field at noninteger flux quantum values arising from the fluxoid quantization, the so-called Little-Parks effect $[16,17]$.

It has been theoretically demonstrated that a stable vortex configuration can be achieved using a SC pinning landscape [18]. In this case, SC dots contribute to the pinning

Published by the American Physical Society under the terms of the Creative Commons Attribution 3.0 License. Further distribution of this work must maintain attribution to the author(s) and the published article's title, journal citation, and DOI. through two mechanisms: (i) structural defects due to the corrugation and (ii) diamagnetic contribution of the dots in the $\mathrm{SC}$ state. The latter distinguishes the pinning mechanisms of $\mathrm{SC}$ pinning centers from normal or magnetic pinning centers. However, little experimental work has been done in exploring SC pinning landscapes [19,20]. In Ref. [19] periodic square arrays of $\mathrm{Nb}$ cylinders were used as pinning centers for $\mathrm{Nb}$. These SC pinning centers are found to be repulsive for the SC vortices. While these studies did not observe clear matching resistance minima, the slope of $R(H)$ changed at matching fields, i.e., where the number of vortices was an integer or half integer times the number of protruding cylinders. Subsequent work reported [20] on the vortex dynamics of $\mathrm{Nb}$ thin films containing a dense triangular array of submicron $\mathrm{V}$ dots, in which the $T_{C}\left(\mathrm{Nb}\right.$ film) was larger than the $T_{C}$ (V dots). In this case, the ac susceptibility shows an abrupt change in vortex mobility across the expected superconducting transition of the $\mathrm{V}$ dots. The vortex lattice responds with lower mobility when the $\mathrm{V}$ dots are in the $\mathrm{SC}$ state. Due to the low superconducting critical field of the $\mathrm{V}$ dots, this behavior could only be observed in a limited field region. While these works show the pinning properties of SC dots and suggest the possibility of commensurability effects by a SC pinning landscape, they do not probe the pinning strength nor do they provide direct evidence of the superconducting character of the dots. We propose an approach in which the $T_{C}$ of the SC film is lower than the $T_{C}$ of the pinning centers which allows investigating the effectiveness of pinning landscape in a wider range of temperature and applied fields.

In this work we present a study on superconducting $\mathrm{V}$ films with periodic superconducting $\mathrm{Nb}$ nanodot arrays, in which the pinning centers have higher $T_{C}$ than the SC film. We observe clear commensurability effects due to SC pinning and we compare it with an equivalent sample with nonsuperconducting $(\mathrm{Cu})$ nanodots. We show that $\mathrm{Nb}$ pinning centers are more effective than $\mathrm{Cu}$ pinning centers, with $\sim 30 \%$ higher critical current density $\left(J_{C}\right)$ at low temperatures. However, the temperature dependence of the superconducting 
properties of the $\mathrm{V}$ film is unexpected. The critical field $\left(H_{C 2}\right)$ and $J_{C}$ of $\mathrm{V}$ on $\mathrm{SC}$ dots drop below the values of $\mathrm{V}$ on nonsuperconducting dots at $T>3 \mathrm{~K}$. We relate this decrease to the crossover between the coherence length $[\xi(T)]$ and the edge-to-edge separation between the dots.

\section{EXPERIMENTAL DETAILS}

We compared the properties of three different samples: (1) "Nb-down," consisting of an array of superconducting $\mathrm{Nb}$ dots with a V film on top; (2) "Cu-down," consisting of an array of nonsuperconducting $\mathrm{Cu}$ dots with a $\mathrm{V}$ film on top; and (3) "Nb-up," consisting of an array of $\mathrm{Nb}$ dots deposited directly on top of a V film. Samples (1) and (2) which are corrugated due to the underlying dots were used to study the role of superconducting versus nonsuperconducting pinning centers. The third sample, $\mathrm{Nb}$-up, was the reference which allows to study and compare the role of corrugation. In addition to these samples we grew the following reference samples: $\mathrm{Nb}$ film $(40 \mathrm{~nm}), \mathrm{V}$ film $(100 \mathrm{~nm})$, and $\mathrm{Nb}(40 \mathrm{~nm}) / \mathrm{V}(100 \mathrm{~nm})$ bilayer.

All samples were grown on $4 \times 4 \mathrm{~mm}^{2}$ highly resistive $(\rho=5000-8000 \Omega \mathrm{cm})$ (100) silicon substrates. V film, $100 \mathrm{~nm}$ thickness, was deposited simultaneously on all samples. Metal evaporation was done by electron beam deposition at a rate of $3 \AA / \mathrm{s}$ in a high-vacuum system with a base pressure of $2 \times 10^{-8}$ Torr.

The coverage of large-area samples with $\mathrm{Nb}$ and $\mathrm{Cu}$ dots was achieved using 300 -nm-thick $\mathrm{Al}_{2} \mathrm{O}_{3}$ porous membranes. These membranes were fabricated from pure $(99.99 \%) \mathrm{Al}$ foil following the two-step anodization method described in Ref. [21]. After anodization, the membranes were transferred onto $\mathrm{Si}$ substrates, for the fabrication of $\mathrm{Nb}$-down and $\mathrm{Cu}$-down, and onto $\mathrm{V}$ thin film for the $\mathrm{Nb}$-up sample. The membrane transferring process was conducted following the method described elsewhere [22]. This approach allows obtaining nanopatterns in large areas and therefore enabling the characterization of magnetic properties by conventional magnetometry techniques.

Figure 1 shows the scanning electron microscopy (SEM) of the $\mathrm{Nb}$ array in the $\mathrm{Nb}$-down sample, prior to $\mathrm{V}$ deposition. The thickness of $\mathrm{Cu}$ and $\mathrm{Nb}$ dots $t=40 \mathrm{~nm}$ was determined from low-angle x-ray reflectivity using continuous reference films. SEM characterization shows that $\mathrm{Nb}$ and $\mathrm{Cu}$ dots are arranged in triangular lattices with identical density and comparable domain size (larger than several microns). Dot diameter $d=70 \mathrm{~nm} \pm 5 \mathrm{~nm}$ and lattice parameter $a=100 \mathrm{~nm} \pm 5 \mathrm{~nm}$ were obtained by quantitative image analysis using the Microscopy Image Segmentation Tool (MIST) software [23].

Electrical transport and magnetization measurements were conducted in a Quantum Design Dynacool system. All temperature-dependent measurements were carried out following a zero-field cool protocol: The samples were cooled to $2 \mathrm{~K}$ at zero magnetic field before the measurements were initiated. In all cases, the applied magnetic field was perpendicular to the sample surface. Magnetization measurements were performed by vibrating sample magnetometry (VSM) as a function of field $( \pm 5 \mathrm{~T})$, and as a function of temperature $(2-9 \mathrm{~K})$. Subsequent magnetoresistance measurements were performed using a four-probe configuration with electrical currents in the range of $1 \mu \mathrm{A}$ to $10 \mathrm{~mA}$. The temperature was set with a pre-

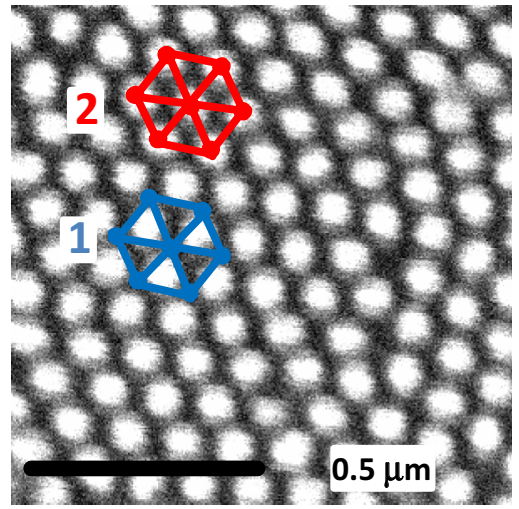

FIG. 1. (Color online) SEM image of triangular array of $\mathrm{Nb}$ dots grown on Si substrate. Drawn hexagons show two different locations for vortices in SC (1, blue line) and non-SC (2, red line) pinning centers.

cision of $\pm 1 \mathrm{mK}$. In addition to these standard measurements, the superconducting transition of $\mathrm{Nb}$ dots was independently determined from magnetic field modulated microwave spectroscopy (MFMMS) [24]. The MFMMS data were obtained with $200-O e$ external dc field, which is modulated by a $15-\mathrm{Oe}$ ac field at $100 \mathrm{kHz}$ and $1 \mathrm{~mW}$ of microwave power.

\section{RESULTS}

The superconducting critical temperature $\left(T_{C}\right)$ and transition width $\left(\Delta T_{C}\right)$ of $\mathrm{Nb}$-down, $\mathrm{Cu}$-down, $\mathrm{Nb}$-up, and the three reference samples: $\mathrm{Nb}$ film $(40 \mathrm{~nm}), \mathrm{V}$ film $(100 \mathrm{~nm})$, and $\mathrm{Nb}(40 \mathrm{~nm}) / \mathrm{V}(100 \mathrm{~nm})$ bilayer (with $\mathrm{V}$ on the top of $\mathrm{Nb}$ ), are shown in Table I. $T_{C}$ values were obtained by resistive measurements, choosing a criterion of $0.8 R_{N}$, where $R_{N}$ is the normal-state resistance. $\mathrm{Nb}$-down and $\mathrm{Cu}$-down samples show wider and multistep transitions, $\Delta T_{C}=0.5$ and $0.4 \mathrm{~K}$, and $T_{C}=4.08$ and $4.33 \mathrm{~K}$, respectively. $\mathrm{Nb}$-up and the reference $\mathrm{V}$ film show the same $T_{C}=4.77 \mathrm{~K}$ and $\Delta T_{C}=0.01 \mathrm{~K}$. The lower $T_{C}$ and larger $\Delta T_{C}$ of the samples with underlying dots can be attributed to the presence of corrugation (film thickness variations) [25] and confinement effects [26]. Since the dot dimensions $(d=70 \mathrm{~nm}$ and $t=40 \mathrm{~nm})$ are comparable to the Bardeen-Cooper-Schrieffer (BCS) coherence lengths$\xi_{\mathrm{o}, \mathrm{Nb}}=38 \mathrm{~nm}$ [27] and $\xi_{\mathrm{o}, \mathrm{V}}=44 \mathrm{~nm}$ [28] for $\mathrm{Nb}$ and $\mathrm{V}$,

TABLE I. Superconducting critical temperature $\left(T_{C}\right)$ and transition width $\left(\Delta T_{C}\right)$ obtained by zero-field resistance measurements. The value of $T_{C}$ was determined by $0.8 R_{N}$ criterion.

\begin{tabular}{lcc}
\hline \hline Sample & $T_{C}(\mathrm{~K})$ & $\Delta T_{C}(\mathrm{~K})$ \\
\hline Nb dots & $6.10^{\mathrm{a}}$ & - \\
Nb film $(40 \mathrm{~nm})$ & 8.00 & 0.01 \\
V film $(100 \mathrm{~nm})$ & 4.77 & 0.01 \\
$\mathrm{Nb} / \mathrm{V}$ bilayer & 8.10 & 0.21 \\
Nb-down & 4.08 & 0.50 \\
Cu-down & 4.33 & 0.40 \\
Nb-up & 4.77 & 0.01 \\
\hline \hline
\end{tabular}

${ }^{\mathrm{a}} T_{C}$ of $\mathrm{Nb}$ dots was obtained by MFMMS. 

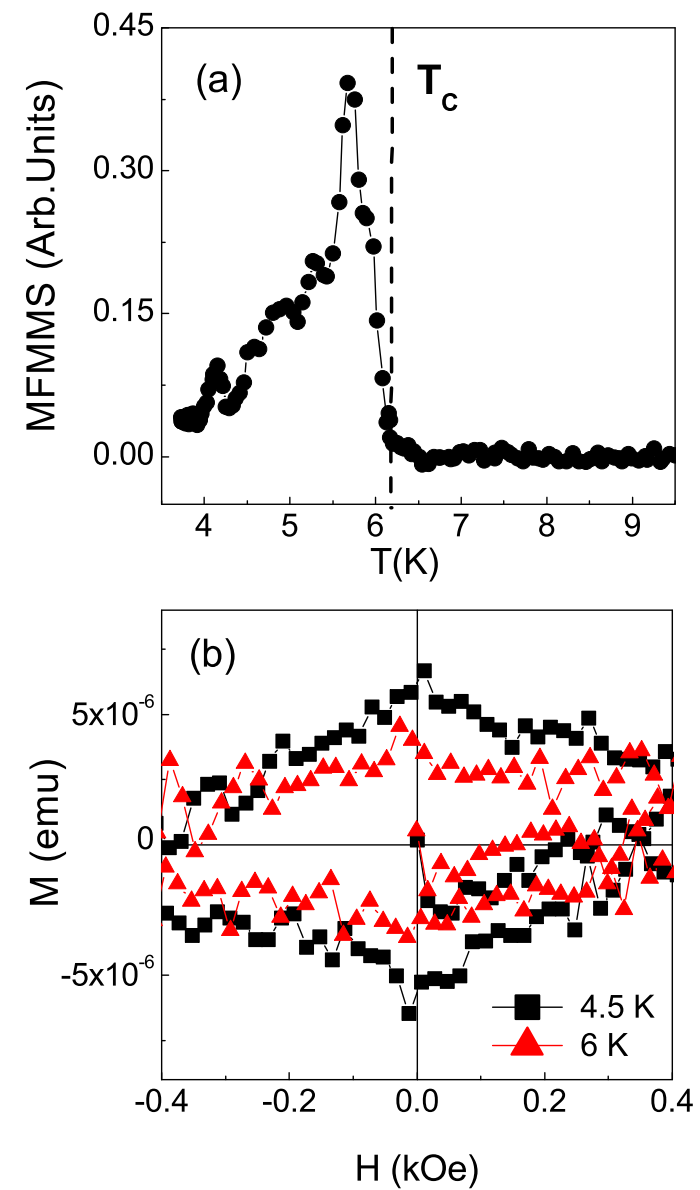

FIG. 2. (Color online) (a) MFMMS signal as a function of temperature with 200-Oe applied dc magnetic field for $\mathrm{Nb}$ dots (sample $\mathrm{Nb}$-down before $\mathrm{V}$ film deposition). (b) $M(H)$ at 4.5 and $6 \mathrm{~K}$ for $\mathrm{Nb}$-down (after $\mathrm{V}$ deposition).

respectively - it is expected that these two effects play an important role. Confinement can also weaken superconductivity in the thinner regions of the corrugated $\mathrm{V}$ film where vortex pinning may occur preferentially.

$\mathrm{Nb}$-up and $\mathrm{V}$-film reference sample have the same $T_{C}$ which confirms that there is no strong proximity coupling between $\mathrm{V}$ film and $\mathrm{Nb}$ dots. This lack of interaction between $\mathrm{Nb}$ and $\mathrm{V}$ can be related to the appearance of an oxide layer which could be formed during the anodic aluminum oxide (AAO) membrane transfer process. Consequently, proximity effects between $\mathrm{Nb}$ dots and $\mathrm{V}$ film are neglected in this paper. In addition, the critical temperatures of $\mathrm{Nb} / \mathrm{V}$ bilayer and $\mathrm{Nb}$-film reference sample are very similar and therefore the superconductivity of $\mathrm{Nb}$ film is not significantly affected by the proximity with $\mathrm{V}$ in the $\mathrm{Nb} / \mathrm{V}$ bilayer.

We measured the $T_{C}$ of $\mathrm{Nb}$ dots by MFMMS at different magnetic fields before V-film deposition. This high-sensitivity technique allows detecting superconductivity in discontinuous systems. Figure 2(a) shows the temperature dependence of the MFMMS signal across the SC transition. In spite of the high microwave power absorption by the Si substrates, the $\mathrm{SC}$ transition peak of $\mathrm{Nb}$ nanodots appears as a peak [24] at $6.1 \mathrm{~K}$. The result shown in Fig. 2(a) was obtained with 200-Oe dc field applied perpendicular to the sample surface.

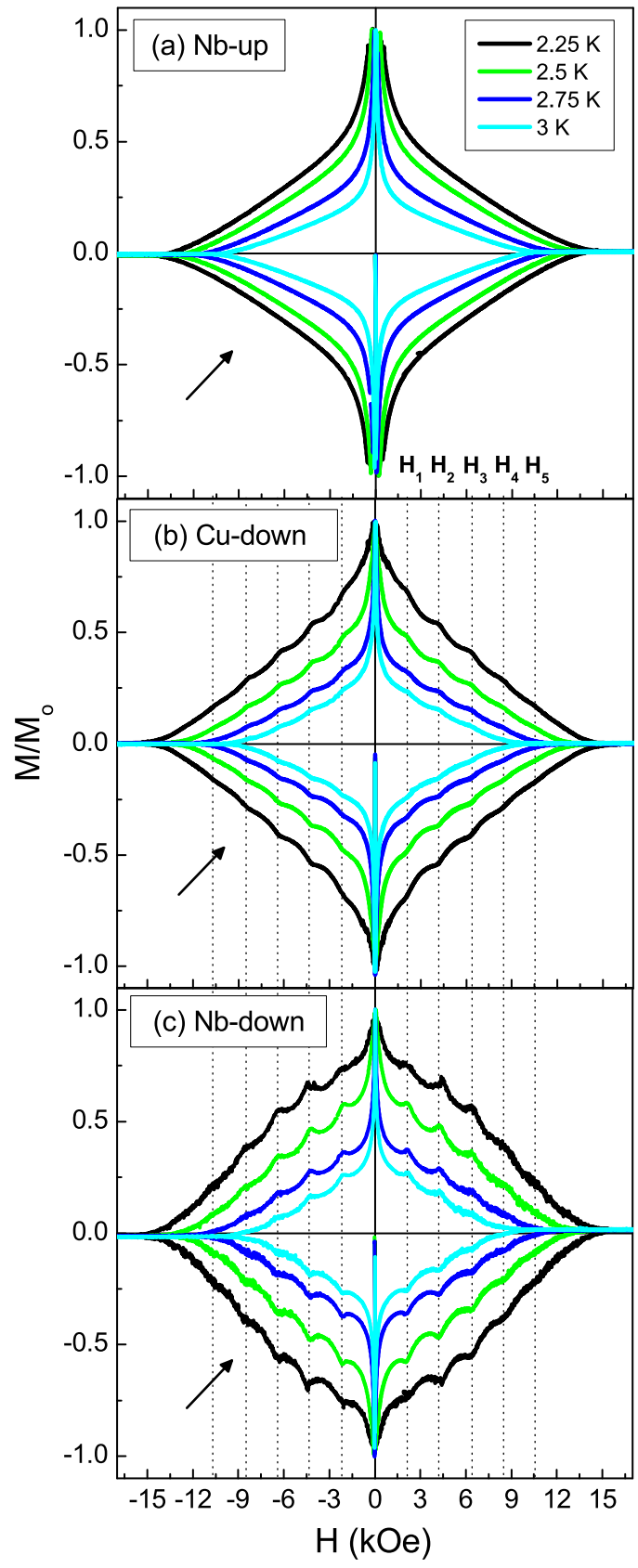

FIG. 3. (Color online) Superconducting loops normalized to maximum magnetization values at different temperatures for (a) $\mathrm{Nb}$-up, (b) Cu-down, and (c) Nb-down. Dashed vertical lines highlight the matching fields at $M(H)$ maxima positions. Arrows point towards increasing temperatures.

No significant change of the SC transition temperature was observed for magnetic fields below 1000-Oe dc field. After the $\mathrm{V}$ film deposition on the $\mathrm{Nb}$ dots (sample $\mathrm{Nb}$-down), we measured magnetization loops above the $T_{C}$ of $\mathrm{V}$, at 4.5 and $6 \mathrm{~K}$. The $M(H)$ loops in Fig. 2(b) confirm the SC character of the $\mathrm{Nb}$ dots after $\mathrm{V}$ deposition. The rather low $T_{C}=6.1 \mathrm{~K}$ for the bare $\mathrm{Nb}$ dots lattice confirms the effect of finite size and lateral confinement in the sample. 
Figure 3 shows the $M(H)$ loops normalized to maximum magnetization of the three samples below $3 \mathrm{~K}$ at the SC state. Prominent peaks up to fifth order can be observed for both $\mathrm{Cu}$-down [Fig. 3(b)] and Nb-down [Fig. 3(c)] samples. These peaks precisely occur at the same field values for both samples. Furthermore, the periodicity of the peaks, $\Delta H=2.12 \pm 0.02 \mathrm{kOe}$, correctly agrees with the matching field values expected for a similar triangular array of dots with a lattice parameter $a=100 \pm 10 \mathrm{~nm}, H_{n}=n \frac{2}{\sqrt{3}} \frac{\emptyset_{0}}{a^{2}}=$ $n(2.39 \pm 0.48) \mathrm{kOe}$, where $\emptyset_{0}=2.07 \times 10^{-15} \mathrm{~Wb}$ is the flux quantum, and $n$ is the matching field order. This indicates that the vortex lattices in both samples, with SC and non-SC pinning centers, are commensurate to the pinning landscape at the same fields, i.e., to the lattice parameter determined by SEM. Note that the initial position of the vortices in relation to the pinning sites, either on vertices or interstitial, does not affect the lattice parameters, i.e., matching field values (see drawn hexagons in Fig. 1). It is also worth noting the lack of matching anomalies in the Nb-up sample which can be attributed to the absence of corrugation in the $\mathrm{V}$ film.

Figure 4 shows the normalized magnetoresistance $R(H) / R_{N}$ used to compare the pinning strength of all three samples close to $T_{C}$. Matching fields are observed in $\mathrm{Cu}$-down [Fig. 4(b)] and Nb-down [Fig. 4(c)] samples at the same field values, which is consistent with magnetization data in Fig. 3. Nb-down shows pronounced minima up to the second order in a wide temperature range, from 3.83 to $4.10 \mathrm{~K}\left(0.94 T_{C}\right.$ to $\left.0.99 T_{C}\right)$, whereas $\mathrm{Cu}$-down shows less pronounced minima only up to the first order in a narrower temperature range, from 3.95 to $4 \mathrm{~K}\left(0.91 T_{C}\right.$ to $\left.0.92 T_{C}\right)$. Furthermore, as expected from the magnetometry [Fig. 3(a)], no matching effects are observed for Nb-up [Fig. 4(a)].

In order to highlight important differences between the three samples, we compare the normalized resistances and magnetization at the same reduced temperature in Fig. 5. The normalized $M(H) / M_{0}$ superconducting loops at $T / T^{*}=0.52$ for $\mathrm{Nb}$-up, $\mathrm{Nb}$ down, $\mathrm{Cu}$-down, and the $\mathrm{Nb} / \mathrm{V}$ bilayer are shown in Fig. 5(a). The Nb-down sample shows stronger superconductivity, evidenced by sharper matching field peaks and larger loop width. We computed the normalized loop widths $\Delta M(H) / M_{0}$ from Fig. 5(a). These widths are proportional to the critical current density, $J_{C}(H)$, as stated by the Bean model [29]. At $T / T^{*}=0.52 \mathrm{~K}, J_{C}$ of Nb-down, evaluated at the second matching field, shows a relative increase of $\sim 30 \%$ with respect to Cu-down, $\sim 120 \%$ with respect to $\mathrm{Nb}$-up, and $\sim 190 \%$ with respect to $\mathrm{Nb} / \mathrm{V}$ bilayer. Figure 5(b) shows $R(H) / R_{N}$ at $T / T^{*}=0.92$ with $T^{*}$ the onset of the superconducting transition for each sample. Close to $T_{C}$, the normalized resistance is higher for the $\mathrm{Nb}$-down than the other two samples. Despite the superconducting character of the $\mathrm{Nb}$ dots, in this temperature range, the superconductivity of $\mathrm{Nb}$-down weakens more with temperature than $\mathrm{Cu}$-down.

In order to understand why the sample with superconducting pinning centers has higher critical current density at low temperature but higher resistance and lower critical temperature, we compared the temperature dependence of the critical fields and the normalized areas of the hysteresis loops of three samples. The inset of Fig. 6 compares the temperature dependence of the critical field $\left(H_{C 2}\right)$ calculated

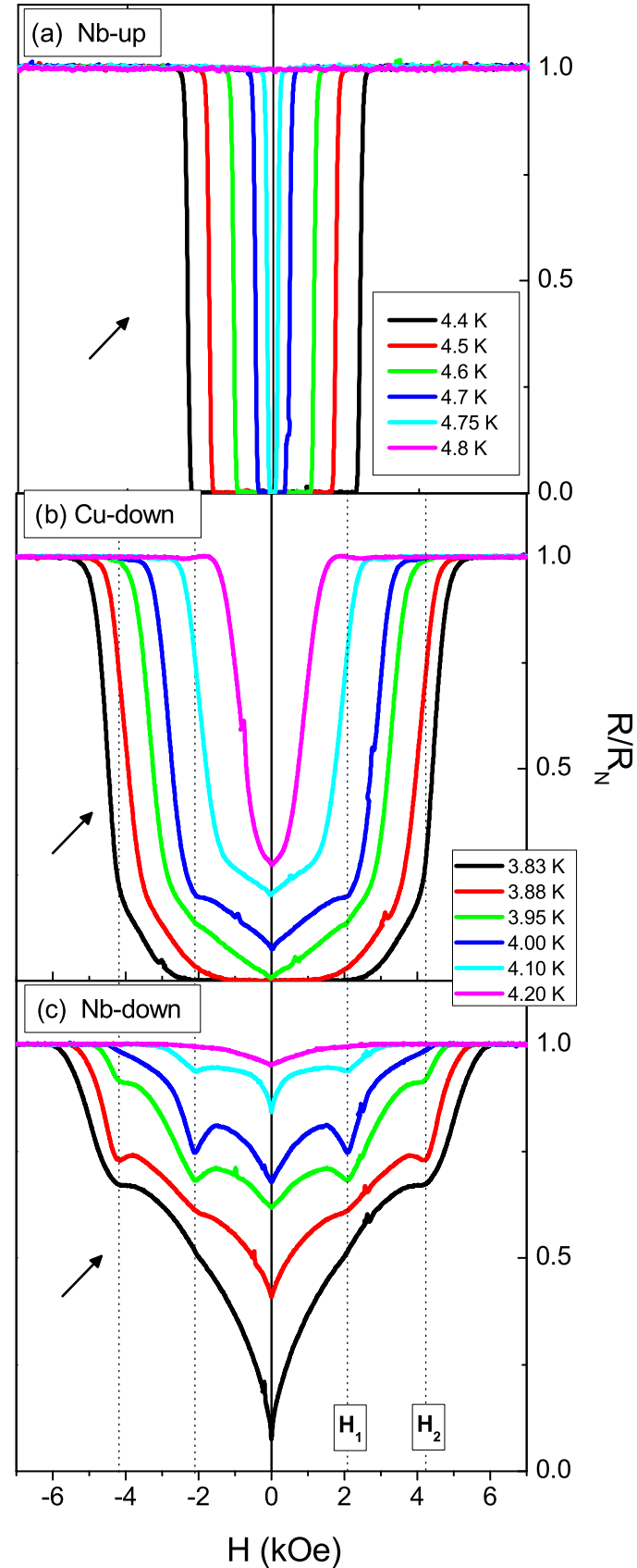

FIG. 4. (Color online) Magnetoresistance normalized to the resistance in the normal state at different temperatures for (a) Nb-up, (b) $\mathrm{Cu}$-down, and (c) Nb-down samples. Dashed vertical lines highlight the matching fields as resistance minima. $H_{1}$ and $H_{2}$ indicate the first two matching fields. Arrows point towards increasing temperatures.

from the loops in Fig. 3 at $M(H)=0$. Below $T=3 \mathrm{~K}, H_{C 2}$ of $\mathrm{Nb}$-down is higher than $\mathrm{Cu}$-down and $\mathrm{Nb}$-up, while it is lower above $T=3 \mathrm{~K}$. Extrapolated $H_{C 2}(T=0 \mathrm{~K})$ values are $36.4,32.9$, and $27.7 \mathrm{kOe}$ for $\mathrm{Nb}$-down, $\mathrm{Cu}$-down, and $\mathrm{Nb}$-up, respectively. Figure 6 shows the total area of $M / M_{o}$ versus $H$ cycles as a function of temperature for the three samples. These normalized hysteresis loop areas are also related to the critical currents since $H_{C 2}$ does not vary significantly between samples [see Fig. 5(a)]. The difference in critical current density of $\mathrm{Nb}$-down and $\mathrm{Cu}$-down decreases with temperature until they 


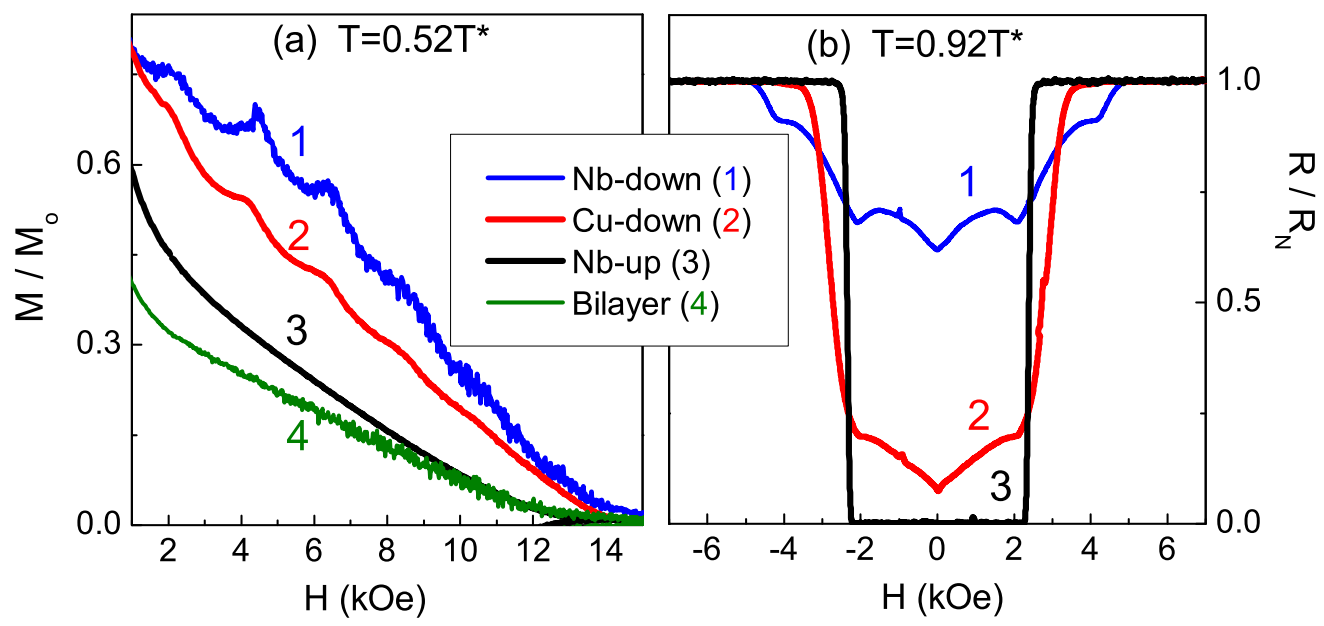

FIG. 5. (Color online) For Nb-down (1, blue line), Cu-down (2, red line), Nb-up (3, black line) and Nb/V bilayer (4, green line): (a) First quadrant of $M(H)$ loops normalized to maximum magnetization values at $T=0.52 T^{*} \mathrm{~K}$. (b) Magnetoresistance normalized to the resistance in the normal state at $T=0.92 T^{*}$.

cross at $T \sim 3 \mathrm{~K}$. This result is consistent with the behavior of the critical field values for all samples.

\section{DISCUSSION}

Although $\mathrm{Nb}$-down has lower $T_{C}$ than $\mathrm{Cu}$-down and $\mathrm{Nb}$-up, we can separate two different temperature regions in which the comparison between the other SC parameters changes gradually. At low temperatures, $T<3 \mathrm{~K}$, the sample with $\mathrm{SC}$ pinning centers (Nb-down) shows more robust superconductivity characterized by sharper matching field peaks, higher critical fields, and larger critical currents (see Figs. 5 and 6). However, as temperature increases, the SC properties of $\mathrm{V}$ on the SC pinning centers ( $\mathrm{Nb}$-down) decrease faster, even though the $\mathrm{Nb}$ dots remain in the $\mathrm{SC}$ state. Above $3 \mathrm{~K}, \mathrm{Nb}$-down shows higher resistance, lower critical currents, and lower critical fields (see Figs. 5 and 6), although it shows well-defined resistive minima up to second order in an unusually wide temperature range [see Fig. 4(c)]. These minima occur at the

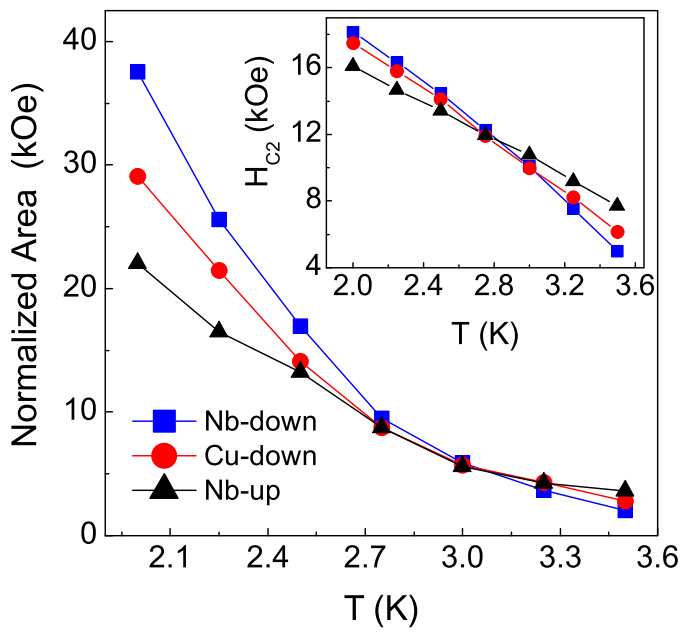

FIG. 6. (Color online) Temperature dependence of the normalized area of the hysteresis loops. Inset: Temperature dependence of $H_{C 2}$ obtained from loops in Fig. 3. expected matching field values, consistent with magnetization data in Fig. 3.

This behavior cannot be attributed to better pinning in non-SC dots, since SC dots show sharper and higher-order resistive minima. We relate this contradictory behavior to a change in the nature of the resistance minima at $3 \mathrm{~K}$. Previous pinning experiments show that when $\xi(T)$ becomes larger than the edge-to-edge distance between pinning centers $(w)$, the nature of resistance minima changes. Minima are no longer related to commensurability of the vortex lattice and the pinning centers [30-32]. They arise from fluxoid quantization, which induces additional $T_{C}$ suppression at noninteger flux quanta [33]. This behavior is known as the superconducting wire network regime.

For the three samples, $\xi(T)=\left(\frac{\emptyset_{0}}{2 \pi H_{C 2}(T)}\right)^{1 / 2}$ was calculated from the temperature dependence of the upper critical field depicted in the inset of Fig. 6 for $T \leqslant 3.5 \mathrm{~K}$ and from the upper critical field measured by transport for $T>3.8 \mathrm{~K}$. In the latter, $H_{C 2}$ was defined at $80 \%$ of the normal-state resistance in the superconducting transition measured with different applied fields. Figure 7 shows the temperature dependence of $\xi(T)\left[\xi(T) \sim \xi(0)\left(1-T / T_{C}\right)^{-1 / 2}\right]$ for the three samples. The extrapolated zero-temperature $\xi(0)$ are 9.52, 10, and $10.91 \mathrm{~nm}$ for $\mathrm{Nb}$-down, $\mathrm{Cu}$-down, and $\mathrm{Nb}$-up, respectively. The horizontal dotted line in Fig. 7 indicates the temperature at which the vortex core diameter $[\sim 1.84 \times \xi(T)]$ becomes comparable to the edge-to-edge separation between the dots $(w \sim 30 \mathrm{~nm})$. The crossover at which $1.84 \times \xi \sim w=30 \mathrm{~nm}$ coincides with the temperature where $H_{C 2}$ and $J_{C}$ of $\mathrm{Nb}$-down and $\mathrm{Cu}$-down samples cross $(T \sim 3 \mathrm{~K})$.

In addition, one of the consequences of the SC network regime is the appearance of $H_{C 2}$ oscillations [16,17] with the same field period as that observed in the $R(H)$ curves. The inset of Fig. 7 shows the nonlinear temperature dependence of $H_{C 2}$ for $\mathrm{Nb}$-down in which a peak at the first matching field, $H_{1}=2.08 \mathrm{kOe}$, is superimposed. This curve suggests the change to a $\mathrm{SC}$ network regime near $T_{C}$.

It has been shown that in samples with non-SC pinning centers, vortices pin on top of the dots, and in interstitial 


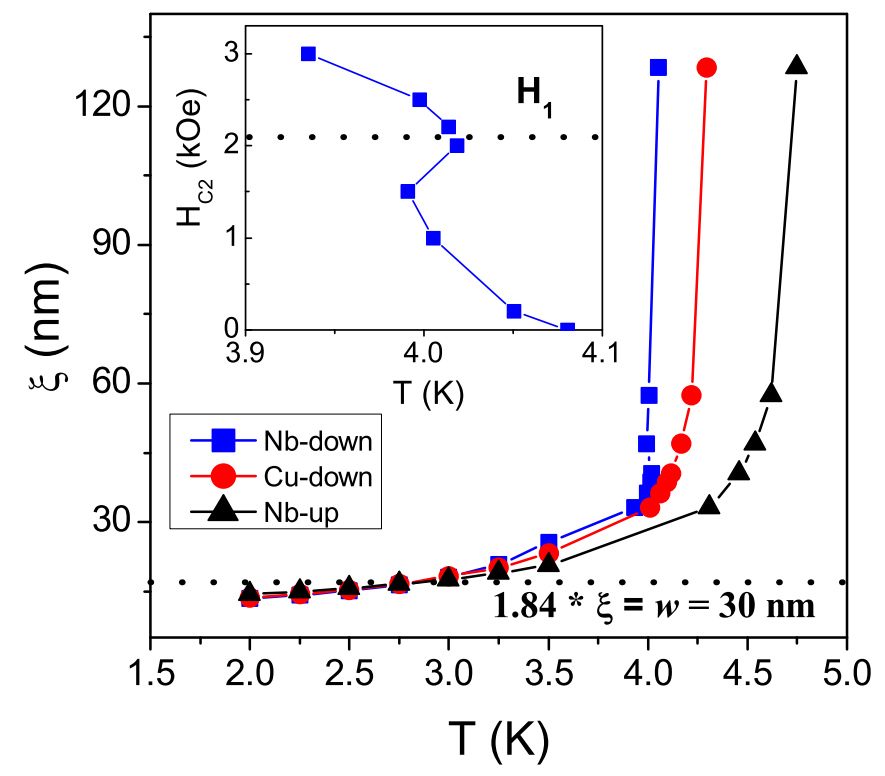

FIG. 7. (Color online) Temperature dependence of coherence length for $\mathrm{Nb}$-down (blue squares), $\mathrm{Cu}$-down (red circles), and $\mathrm{Nb}$-up (black triangles). Inset: Temperature dependence of $H_{C 2}$ for Nb-down.

positions depending on the external applied magnetic field. When the applied field reaches $H=H_{s}=n_{s} H_{1}$, where $n_{s}$ is the "saturation number," an additional flux line penetrating the film will be repelled into the interstitial region between the dots [34]. Interstitial vortices are only stable due to caging between vortices [6,35]. On the contrary, it is accepted [18] that SC pinning centers pin vortices in interstitial positions. This is due to the diamagnetic repulsion between vortices and SC pinning sites. Based on these results, we assume that in $\mathrm{Cu}$-down, vortices pin on top of the attractive pinning centers and in interstitial positions. Note that for $T<3 \mathrm{~K}$, $n_{s}=\frac{d}{4 \xi(T)} \sim 1$ and the maximum number of vortices per pinning center derived from Fig. 3 is $n_{\max }=5$. In Nb-down, the vortices pin preferentially in interstitial positions. SEM images of samples similar to the ones described here indicate that the superconducting layer on top of the nanodots is thinner between dots and thicker on top of the dots. In addition the low ratio $a / d \sim 1$ corresponds to the case of thin superconducting interstices of width $a-d \sim \xi_{o}$ [36]. These results support our point; corrugation and confinement effects weaken the superconductivity in interstitial positions. Therefore these regions act as preferential pinning centers. At low temperatures, the repulsive interactions exerted by the SC pinning sites assist pinning by caging the vortices at interstitial positions. However, at higher temperatures, the interaction between SC pinning sites and flux lines weaken the superconductivity when the superconducting regime changes from pinning enhancement to SC wire network in which fluxoids spread over several dot lattice cells. This vortex regime change explains the overall increase in the resistance and the crossover in the temperature dependence of the critical field and critical current of $\mathrm{Nb}$-down and $\mathrm{Cu}$-down samples at $T=3 \mathrm{~K}$.

The previous argument also explains the lack of pinning in samples without corrugation, i.e., $\mathrm{Nb}$-up sample. Corrugation is still the main pinning mechanism, while the repulsive forces between the diamagnetism of the pinning centers and the vortices strengthens the pinning at low temperature, but it is not enough to lock the vortex lattice by itself.

However, further studies on the interaction between vortices and pinning centers across the transition to the $\mathrm{SC}$ wire network regime are necessary to support the conclusions. In order to obtain more information on the processes behind the observed decrease of the SC thin film properties, we suggest that a study of this process using different dot widths and lattice parameters is required. Ideally one would choose two materials that fulfill $T_{C \text {,dots }}>T_{C \text {,film }}$ and $H_{C 2 \text {,dots }}<H_{C 2 \text {,film }}$. This system would allow tuning the SC state of the dots by applying an external field, while the film is in the wire network regime. If superconductivity of the dots is related to the decrease of the superconducting properties of the thin film, a sudden improvement of them is expected when the dots become a normal metal.

\section{CONCLUSIONS}

We presented a systematic study on the role of superconducting pinning centers and corrugation on the pinning in the superconducting film. The corrugation decreases the critical temperature of the film, but it is necessary for pinning. SC pinning centers strengthen the pinning, increasing the sharpness and depth of resistance minima and the width of magnetization loops at low temperatures (below $3 \mathrm{~K}$ ). However, these behaviors show unexpected temperature dependence and the presence of SC pinning centers induces a faster decrease of the SC properties above $3 \mathrm{~K}$. The $\mathrm{V}$ thin film $\left(T_{C} \sim 4.5 \mathrm{~K}\right)$ with $\mathrm{Nb}$ pinning centers $\left(T_{C} \sim 6.1 \mathrm{~K}\right)$ has a higher resistance, lower critical field, lower critical temperature, and lower critical current than a $\mathrm{V}$ thin film with $\mathrm{Cu}$ pinning centers. This effect is related to a change in the nature of the resistance minima when the edge-to-edge distance between pinning centers is of the order of the vortex core diameter.

\section{ACKNOWLEDGMENTS}

We thank J. Wampler for help with the fabrications of porous alumina masks. The sample preparation and characterization was supported by the U.S. AFOSR Grant No. FA955014-1-0202. Work was supported by Spanish MINECO under Grant No. FIS2013-45469 and CAM Grant No. S2013/MIT2850. E.N. acknowledges support from Ministerio de Educación, Cultura y Deporte, Subprograma Estatal de Movilidad, Salvador de Madariaga 2014.
[1] A. I. Larkin and Yu. N. Ovchinnikov, J. Low Temp. Phys. 34, 409 (1979).
[2] A. Hoffmann, P. Prieto, and I. K. Schuller, Phys. Rev. B 61, 6958 (2000). 
[3] C. Reichhardt and C. J. Olson Reichhardt, Phys. Rev. B 76, 094512 (2007).

[4] S. Guénon, Y. J. Rosen, Ali C. Basaran, and I. K. Schuller, Appl. Phys. Lett. 102, 252602 (2013); D. Ray, C. J. Olson Reichhardt, B. Jankó, and C. Reichhardt, Phys. Rev. Lett. 110, 267001 (2013).

[5] A. T. Fiory, A. F. Hebard, and S. Somekh, Appl. Phys. Lett. 32, 73 (1978).

[6] K. Harada, O. Kamimura, H. Kasai, T. Matsuda, A. Tonomura, and V. V. Moshchalkov, Science 274, 1167 (1996).

[7] V. Metlushko, U. Welp, G. W. Crabtree, R. Osgood, S. D. Bader, L. E. DeLong, Z. Zhang, S. R. J. Brueck, B. Ilic, K. Chung, and P. J. Hesketh, Phys. Rev. B 60, R12585 (1999).

[8] G. R. Berdiyorov, M. V. Miloševic, and F. M. Peeters, Phys. Rev. Lett. 96, 207001 (2006); Phys. Rev. B 74, 174512 (2006).

[9] A. Bezryadin, Yu. N. Ovchinnikov, and B. Pannetier, Phys. Rev. B 53, 8553 (1996).

[10] S. Raedts, A. V. Silhanek, M. J. Van Bael, and V. V. Moshchalkov, Phys. Rev. B 70, 024509 (2004).

[11] J. I. Martín, M. Vélez, J. Nogués, and I. K. Schuller, Phys. Rev. Lett. 79, 1929 (1997).

[12] M. Lange, M. J. Van Bael, Y. Bruynseraede, and V. V. Moshchalkov, Phys. Rev. Lett. 90, 197006 (2003); M. V. Milošević and F. M. Peeters, Europhys. Lett. 70, 670 (2005).

[13] Y. Jaccard, J. I. Martín, M.-C. Cyrille, M. Vélez, J. L. Vicent, and I. K. Schuller, Phys. Rev. B 58, 8232 (1998).

[14] M. J. Van Bael, K. Temst, V. V. Moshchalkov, and Y. Bruynseraede, Phys. Rev. B 59, 14674 (1999).

[15] X. S. Ling, H. J. Lezec, M. J. Higgins, J. S. Tsai, J. Fujita, H. Numata, Y. Nakamura, Y. Ochiai, Chao Tang, P. M. Chaikin, and S. Bhattacharya, Phys. Rev. Lett. 76, 2989 (1996).

[16] R. D. Parks and W. A. Little, Phys. Rev. 133, A97 (1964)

[17] B. Pannetier, J. Chaussy, R. Rammal, and J. C. Villegier, Phys. Rev. Lett. 53, 1845 (1984).

[18] G. R. Berdiyorov, V. R. Misko, M. V. Miloševic, W. Escoffier, I. V. Grigorieva, and F. M. Peeters, Phys. Rev. B 77, 024526 (2008).
[19] J. I. Facio, A. Abate, J. Guimpel, and P. S. Cornaglia, J. Phys.: Condens. Matter 25, 245701 (2013).

[20] S. J. Carreira, C. Chiliotte, V. Bekeris, Y. J. Rosen, C. Monton, and I. K. Schuller, Supercond. Sci. Technol. 27, 085007 (2014).

[21] C.-P. Li, I. V. Roshchin, X. Batlle, M. Viret, F. Ott, and I. K. Schuller, J. Appl. Phys. 100, 074318 (2006).

[22] J. E. Allen, K. G. Yager, H. Hlaing, C. Y. Nam, B. M. Ocko, and C. T. Black, Appl. Phys. Lett. 99, 163301 (2011).

[23] I. Valmianski, C. Monton, and I. K. Schuller, Rev. Sci. Instrum. 85, 033701 (2014).

[24] J. G. Ramírez, A. C. Basaran, J. de la Venta, J. Pereiro, and I. K. Schuller, Rep. Prog. Phys. 77, 093902 (2014).

[25] M. I. Montero, J. J. Akerman, A. Varilci, and I. K. Schuller, Europhys. Lett. 63, 118 (2003).

[26] S. Bose, P. Raychaudhuri, R. Banerjee, P. Vasa, and P. Ayyub, Phys. Rev. Lett. 95, 147003 (2005).

[27] C. Kittel, Introduction to Solid State Physics, 7th ed. (Wiley, New York, 1996).

[28] I. A. Garifullin, N. N. Garif'yanov, R. I. Salikhov, and L. R. Tagirov, JETP Lett. 87, 316 (2008).

[29] C. P. Bean, Phys. Rev. Lett. 8, 250 (1962); Rev. Mod. Phys. 36, 31 (1964).

[30] U. Patel, Z. L. Xiao, J. Hua, T. Xu, D. Rosenmann, V. Novosad, J. Pearson, U. Welp, W. K. Kwok, and G. W. Crabtree, Phys. Rev. B 76, 020508(R) (2007).

[31] V. V. Moshchalkov, M. Baert, V. V. Metlushko, E. Rosseel, M. J. Van Bael, K. Temst, Y. Bruynseraede, and R. Jonckheere, Phys. Rev. B 57, 3615 (1998).

[32] U. Welp, Z. L. Xiao, J. S. Jiang, V. K. Vlasko-Vlasov, S. D. Bader, G. W. Crabtree, J. Liang, H. Chik, and J. M. Xu, Phys. Rev. B 66, 212507 (2002).

[33] M. Tinkham, D. W. Abraham, and C. J. Lobb, Phys. Rev. B 28, 6578 (1983).

[34] V. V. Metlushko, L. E. DeLong, V. V. Moshchalkov, and Y. Bruynseraede, Physica C 391, 196 (2003).

[35] C. Reichhardt and C. J. Olson Reichhardt, Phys. Rev. B 79, 134501 (2009).

[36] L. E. De Long, S. Kryukov, V. V. Metlushko, V. V. Moshchalkov, and Y. Bruynseraede, Physica C 404, 123 (2004). 